\title{
New physics in $B$ meson mixing: Future sensitivity and limitations
}

\author{
Jérôme Charles $\odot,{ }^{1, *}$ Sébastien Descotes-Genon $\odot,{ }^{2, *}$ Zoltan Ligeti $\odot,{ }^{3}$ Stéphane Monteil, ${ }^{4, *}$ Michele Papucci, ${ }^{5}$ \\ Karim Trabelsi®, ${ }^{2, *}$ and Luiz Vale Silva $\odot^{6, *}$ \\ ${ }^{1}$ Aix Marseille Univ, Université de Toulon, CNRS, CPT, F-13288 Marseille, France \\ ${ }^{2}$ Université Paris-Saclay, CNRS/IN2P3, IJCLab, 91405 Orsay, France \\ ${ }^{3}$ Ernest Orlando Lawrence Berkeley National Laboratory, University of California, \\ Berkeley, California 94720, USA \\ ${ }^{4}$ Université Clermont Auvergne, CNRS/IN2P3, LPC, F-63000 Clermont-Ferrand, France \\ ${ }^{5}$ Burke Institute for Theoretical Physics, California Institute of Technology, \\ Pasadena, California 91125, USA \\ ${ }^{6}$ IFIC, Universitat de València-CSIC, Parc Científic, Cat. José Beltrán 2, E-46980 Paterna, Spain
}

(Received 19 June 2020; accepted 28 August 2020; published 28 September 2020)

\begin{abstract}
The mixing of neutral mesons is sensitive to some of the highest scales probed in laboratory experiments. In light of the planned LHCb Upgrade II, a possible upgrade of Belle II, and the broad interest in flavor physics in the tera- $Z$ phase of the proposed FCC-ee program, we study constraints on new physics contributions to $B_{d}$ and $B_{s}$ mixings which can be obtained in these benchmark scenarios. We explore the limitations of this program, and identify the measurement of $\left|V_{c b}\right|$ as one of the key ingredients in which progress beyond current expectations is necessary to maximize future sensitivity. We speculate on possible solutions to this bottleneck. Given the current tension with the standard model (SM) in semileptonic $B$ decays, we explore how its resolution may impact the search for new physics in mixing. Even if new physics has the same Cabibbo-Kobayashi-Maskawa and loop suppressions of flavor changing processes as the SM, the sensitivity will reach $2 \mathrm{TeV}$, and it can be much higher if any SM suppressions are lifted. We illustrate the discovery potential of this program.
\end{abstract}

DOI: 10.1103/PhysRevD.102.056023

\section{INTRODUCTION}

The mixing of neutral mesons has provided severe constraints on new degrees of freedom at high energies: since measurements of mixing and $C P$ violation in neutral kaons in the 1960s, it has provided precious information on charm and top quarks before their discovery. The hypothesis of Kobayashi-Maskawa for the origin of $C P$ violation [1] observed in kaons was only tested experimentally when $B A B A R$ and Belle around 2003-2004 established CP violation in good agreement with the predictions of the standard model (SM) $[2,3]$. These $B$-factory results showed that the standard model (SM) source of $C P$ violation in the flavor sector was the dominant part. However, even after $B A B A R$ and Belle, and the $\mathrm{LHCb}$ results of the last decade, new physics (NP) is still allowed to contribute at the $20 \%-$ $30 \%$ level, compared to the SM, in flavor-changing neutral current (FCNC) processes.

\section{For the CKMfitter Group}

Published by the American Physical Society under the terms of the Creative Commons Attribution 4.0 International license. Further distribution of this work must maintain attribution to the author(s) and the published article's title, journal citation, and DOI. Funded by SCOAP.
Since neutral-meson mixings are FCNC processes which are suppressed in the SM, they provide strong constraints on new physics. This led to the development of numerous mechanisms to suppress such contributions, should NP exist at the $\mathrm{TeV}$ scale. Low-energy supersymmetry is one example, where the ansatz of degeneracy or alignment were both motivated by constraints from neutral meson mixing and other FCNC processes. In a large class of NP models the unitarity of the Cabibbo-Kobayashi-Maskawa (CKM) matrix is maintained, and the most significant NP effects occur in observables that vanish at tree level in the SM [4-7]. In such scenarios, which encompass a large class of models, possible effects of heavy particles in each neutral meson system can be described by two real parameters,

$$
M_{12}=\left(M_{12}\right)_{\mathrm{SM}} \times\left(1+h e^{2 i \sigma}\right),
$$

where $M_{12}$ relates to the time evolution of the two-state neutral meson system (for a review, see [8]). However, the extraction of NP contribution to meson mixing is entangled with the determination of the SM parameters, namely the CKM elements. It is not enough to measure the mixing amplitude itself, only the combination of many measurements can reveal a deviation from the SM. In the SM CKM 
fit $[2,9]$, the constraints come from $\Delta F=1$ processes dominated by tree-level charged-current interactions, and $\Delta F=2$ meson mixing processes, which first arise at oneloop level. We can modify the CKM fit to constrain new physics in $\Delta F=2$ processes, under the assumption that it does not significantly affect the SM tree-level chargedcurrent interactions.

The parametrization in Eq. (1) is convenient because any NP contribution to $M_{12}$ is additive with respect to the SM amplitude, so it is easy to read off from a fit the bounds on the magnitude and the phase of the NP contribution, or to convert the result to bounds on SMEFT operators $[10,11]$. In particular, for the NP contribution to the mixing of a meson with $q_{i} \bar{q}_{j}$ flavor quantum numbers, due to the operator

$$
\frac{C_{i j}^{2}}{\Lambda^{2}}\left(\bar{q}_{i, L} \gamma_{\mu} q_{j, L}\right)^{2}
$$

where $C_{i j}$ is related to the flavor dependence and $\Lambda$ to the NP energy scale, one finds in the cases of $B_{d}$ or $B_{s}$ meson mixing [12]

$$
\begin{aligned}
& h \simeq 1.5 \frac{\left|C_{i j}\right|^{2}}{\left|\lambda_{i j}^{t}\right|^{2}} \frac{(4 \pi)^{2}}{G_{F} \Lambda^{2}} \simeq \frac{\left|C_{i j}\right|^{2}}{\left|\lambda_{i j}^{t}\right|^{2}}\left(\frac{4.5 \mathrm{TeV}}{\Lambda}\right)^{2}, \\
& \sigma=\arg \left(C_{i j} \lambda_{i j}^{t *}\right),
\end{aligned}
$$

where $\lambda_{i j}^{t}=V_{t i}^{*} V_{t j}$ and $V$ is the CKM matrix. Operators of different chiralities have conversion factors differing by $\mathcal{O}(1)$ factors [13]. Minimal flavor violation (MFV), where the NP contributions are aligned with the SM ones, correspond to $\sigma=0(\bmod \pi / 2)$.

Substantial recent developments make it interesting to revisit the expected future sensitivities derived in 2013 [12], and to explore if there are any limitations to improve the sensitivity to higher scales, from constraining NP contributions to neutral meson mixing. The LHCb Upgrade II has been proposed [14] and is likely to proceed, and discussions on a possible upgrade of Belle II have started [15]. Moreover, the FCC-ee phase of a future circular collider as a tera- $Z$ factory is generating much interest, due to the versatility of the machine center-of-mass energy [16], which allows the study of all relevant electroweak thresholds $(Z, W W, Z H$, and $t \bar{t})$ and addresses electroweak precision physics (Higgs physics, electroweak precision observables at $Z$ pole and $W W$ thresholds) in an unrivaled way, benefiting simultaneously from both the statistics and the exquisite measurement of the beam energy at the $Z$ and $W W$ thresholds. This physics case is complemented by the unprecedented statistics attainable at the $Z$ pole $\left(\mathcal{O}\left(5 \times 10^{12}\right) Z\right.$ decays $)$ that can be used for flavor physics measurements, exploiting the clean experimental environment (similar to Belle II), and the production of all species of heavy flavors and the large boost (similar to LHCb).
This paper considers thus the following future "phases," as benchmarks to study:

(i) Phase I: LHCb 50/fb, Belle II 50/ab (late 2020s);

(ii) Phase II: LHCb 300/fb, Belle II 250/ab (late 2030s);

(iii) Phase III: Phase II + FCC-ee $\left(5 \times 10^{12} \mathrm{Z}\right.$ decays $)$.

The "Phase I" benchmark here coincides with "Stage II" in Ref. [12], and can be seen as an update of that projection. These data are expected to be collected by the late 2020 s. The "Phase II" benchmark reflects the well-developed case of the LHCb Upgrade II [14] and a possible upgrade of Belle II, which starts being discussed [15]. These data sets may be collected by the late 2030s. Phase III corresponds to a future circular $e^{+} e^{-}$collider collecting $5 \times 10^{12} \mathrm{Z}$ decays. (Order $10^{9}-10^{10} Z$ decays would not reach sensitivities to generic new physics in $B$ decays beyond Phase II.)

We will focus on $B_{d}$ and $B_{s}$ mixing, and do not consider $K$ and $D$ mixing in this paper. For $K$ mixing, it is most natural to parameterize NP via an additive term to the socalled $t t$ contribution to $M_{12}^{K}$ in the SM. To fully constrain its magnitude and phase two observables are needed, $\epsilon_{K}$ and $\Delta m_{K}$. However, the $t t$ contribution is only a small part of the SM contribution to $\Delta m_{K}$, so large reductions in lattice QCD uncertainties would be needed for meaningful improvements compared to Ref. [12]. Regarding $D$-meson mixing, the data may be accommodated by long-distance SM contributions; nevertheless important constraints on NP exist from requiring that NP contributions should not be much larger than the measurements.

In the following, Sec. II discusses the fits, starting from their inputs in Sec. II A. Section II B discusses the current status, while Sec. IIC, II D, IIE contain the results for Phases I, II, and III, respectively. Section III discusses interpretations. Section IV explores future limitations and possible ideas that may lead to improved measurements compared to current expectations. We also explore scenarios in which NP contributes to charged current $b \rightarrow c, u$ transitions, as hinted at by the $3 \sigma$ tension in measurements of the so-called $R(D)$ and $R\left(D^{*}\right)$ ratios of semileptonic rates [17]. Section V concludes.

\section{FITTING THE $B_{d, s}$ MIXING AMPLITUDES}

\section{A. Inputs}

We follow the CKMfitter approach for the CKM global fit $[2,9,18,19]$ with its extension to NP in $\Delta F=2[2,12,20-22]$ (for other studies of such NP, see Refs. [3,23-27]). We fit at the same time the CKM parameters and the NP parameters, using all the inputs available with a well-controlled sensitivity to the CKM and NP parameters.

Table I shows all inputs and their uncertainties considered in our fits. For an easier comparison with the present status as of Summer 2019 [28], the column "Current" shows the current uncertainties (with uncertainties 
TABLE I. Central values and uncertainties used in our analysis. Central values have been adjusted to eliminate tensions when moving to the smaller uncertainties typical of the future projections. The entries "id" refer to the value in the same row in the previous column. The assumptions entering Phase I, Phase II and Phase III estimates are described in the text.

\begin{tabular}{|c|c|c|c|c|c|c|}
\hline & \multirow{2}{*}{$\begin{array}{c}\text { Central } \\
\text { values }\end{array}$} & \multicolumn{4}{|c|}{ Uncertainties } & \multirow{2}{*}{$\begin{array}{l}\text { Reference } \\
\text { Phases I-III }\end{array}$} \\
\hline & & Current [28] & Phase I & Phase II & Phase III & \\
\hline$\left|V_{u d}\right|$ & 0.97437 & \pm 0.00021 & id & id & id & [28] \\
\hline$\left|V_{u s}\right| f_{+}^{K \rightarrow \pi}(0)$ & 0.2177 & \pm 0.0004 & id & id & id & {$[28]$} \\
\hline$\left|V_{c d}\right|$ & 0.2248 & \pm 0.0043 & \pm 0.003 & id & id & {$[40,41]$} \\
\hline$\left|V_{c s}\right|$ & 0.9735 & \pm 0.0094 & id & id & id & {$[28,40,41]$} \\
\hline$\Delta m_{d}\left[\mathrm{ps}^{-1}\right]$ & 0.5065 & \pm 0.0019 & id & id & id & [17] \\
\hline$\Delta m_{s}\left[\mathrm{ps}^{-1}\right]$ & 17.757 & \pm 0.021 & id & id & id & [17] \\
\hline$\left|V_{c b}\right|_{\mathrm{SL}} \times 10^{3}$ & 42.26 & \pm 0.58 & \pm 0.60 & \pm 0.44 & id & [29] \\
\hline$\left|V_{c b}\right|_{W \rightarrow c b} \times 10^{3}$ & 42.26 & & $\ldots$ & & \pm 0.17 & [34-36] \\
\hline$\left|V_{u b}\right|_{\mathrm{SL}} \times 10^{3}$ & 3.56 & \pm 0.22 & \pm 0.042 & \pm 0.032 & id & [29] \\
\hline$\left|V_{u b} / V_{c b}\right|\left(\right.$ from $\left.\Lambda_{b}\right)$ & 0.0842 & \pm 0.0050 & \pm 0.0025 & \pm 0.0008 & id & {$[30]$} \\
\hline $\mathcal{B}(B \rightarrow \tau \nu) \times 10^{4}$ & 0.83 & \pm 0.24 & \pm 0.04 & \pm 0.02 & \pm 0.009 & {$[29,34]$} \\
\hline $\mathcal{B}(B \rightarrow \mu \nu) \times 10^{6}$ & 0.37 & $\ldots$ & \pm 0.03 & \pm 0.02 & id & [29] \\
\hline $\sin 2 \beta$ & 0.680 & \pm 0.017 & \pm 0.005 & \pm 0.002 & \pm 0.0008 & {$[29,30,34]$} \\
\hline$\alpha\left[^{\circ}\right]\left(\bmod 180^{\circ}\right)$ & 91.9 & \pm 4.4 & \pm 0.6 & id & id & [29] \\
\hline$\gamma\left[^{\circ}\right]\left(\bmod 180^{\circ}\right)$ & 66.7 & \pm 5.6 & \pm 1 & \pm 0.25 & \pm 0.20 & {$[29,30,34]$} \\
\hline$\beta_{s}[\mathrm{rad}]$ & -0.035 & \pm 0.021 & \pm 0.014 & \pm 0.004 & \pm 0.002 & {$[30,34]$} \\
\hline$A_{\mathrm{SL}}^{d} \times 10^{4}$ & -6 & \pm 19 & \pm 5 & \pm 2 & \pm 0.25 & {$[14,17,34,37]$} \\
\hline$A_{\mathrm{SL}}^{s} \times 10^{5}$ & 3 & \pm 300 & \pm 70 & \pm 30 & \pm 2.5 & {$[14,17,34,37]$} \\
\hline $\bar{m}_{t}[\mathrm{GeV}]$ & 165.30 & \pm 0.32 & id & id & \pm 0.020 & {$[28,34]$} \\
\hline$\alpha_{s}\left(m_{Z}\right)$ & 0.1185 & \pm 0.0011 & id & id & \pm 0.00003 & {$[28,34]$} \\
\hline$f_{+}^{K \rightarrow \pi}(0)$ & 0.9681 & \pm 0.0026 & \pm 0.0012 & id & id & [30] \\
\hline$f_{K}[\mathrm{GeV}]$ & 0.1552 & \pm 0.0006 & \pm 0.0005 & id & id & [30] \\
\hline$f_{B_{s}}[\mathrm{GeV}]$ & 0.2315 & \pm 0.0020 & \pm 0.0011 & id & id & [30] \\
\hline$B_{B_{s}}$ & 1.219 & \pm 0.034 & \pm 0.010 & \pm 0.007 & id & [30] \\
\hline$f_{B_{s}} / f_{B_{d}}$ & 1.204 & \pm 0.007 & \pm 0.005 & id & id & [30] \\
\hline$B_{B_{s}} / B_{B_{d}}$ & 1.054 & \pm 0.019 & \pm 0.005 & \pm 0.003 & id & [30] \\
\hline$\tilde{B}_{B_{s}} / \tilde{B}_{B_{d}}$ & 1.02 & \pm 0.05 & \pm 0.013 & id & id & {$[30,42,43]$} \\
\hline$\tilde{B}_{B_{s}}$ & 0.98 & \pm 0.12 & \pm 0.035 & id & id & {$[30,42,43]$} \\
\hline$\eta_{B}^{D_{s}}$ & 0.5522 & \pm 0.0022 & id & id & id & [44] \\
\hline
\end{tabular}

combined in quadrature, while in our Summer 2019 analysis statistical and theoretical uncertainties are distinguished). We use standard SM notation for the inputs, even for quantities which may be affected by NP in $\Delta F=2$ processes, whose measurements have to be reinterpreted to include the NP contributions (e.g., $\alpha, \beta, \beta_{s}$ ). Considering the difficulty to ascertain the breakdown between statistical and systematic uncertainties in theoretical inputs for the future projections, for simplicity, we treat all future uncertainties as Gaussian, except for $\eta_{B}$ and $\alpha_{s}\left(m_{Z}\right)$ that we treat in the Rfit model [9].

The extrapolation of lattice QCD inputs is a delicate task, since some of these results are already dominated by systematics that cannot be scaled easily over time. Lattice QCD inputs are taken from Refs. [29,30], with most instances in Table I coming from the latter (in Sec. IID we comment on the impact of using the mixing parameters in Ref. [29]). We are not aware of estimates of lattice
QCD uncertainties that go farther into the future than these. The predicted lattice QCD improvements will be very important for the bag parameters related to the mixing matrix elements, $\left\langle\bar{B}_{q}\left|\left(\bar{b}_{L} \gamma_{\mu} q_{L}\right)^{2}\right| B_{q}\right\rangle=(2 / 3) m_{B_{q}}^{2} f_{B_{q}}^{2} B_{B_{q}}$. Due to the chiral extrapolations to light quark masses, more accurate results are available for matrix elements involving the $B_{s}$ meson, or for ratios between $B_{d}$ and $B_{s}$ hadronic inputs, compared to the results for $B_{d}$ matrix elements. This motivated our choice of lattice inputs in Table I.

The projections for the uncertainties of the exclusive semileptonic determinations of $\left|V_{c b}\right|$ and $\left|V_{u b}\right|$ combine statistical and theoretical uncertainties, the latter coming from lattice QCD extractions of the relevant form factors [29]. For Phase I, we use the predictions labeled "10 yr w/EM," which should be conservative, not assuming that electromagnetic corrections are fully calculated on the lattice. For Phase II, we use the prediction 
labeled "10 yrw/oEM," assuming that electromagnetic corrections will have been computed. The best determinations of $\left|V_{c b}\right|$ until Phase II come from semileptonic $B$ decays, whereas in Phase III from $W \rightarrow \bar{b} c$. For $\left|V_{u b}\right|$, around Phase II, its determination from $B \rightarrow \tau \bar{\nu}$ may become competitive with semileptonic decays.

The main uncertainties in the constraints on $\bar{\rho}$ and $\bar{\eta}$ come from the tree-level inputs $\gamma$ and $\left|V_{u b} / V_{c b}\right|$. The combination of measurements $\gamma(\alpha)=\pi-\beta-\alpha$, which is not affected by NP in $\Delta F=2$ [4], is significant in the current average of the $\gamma$ constraint, but it diminishes in importance at Phase I and especially beyond that (the determination of $\alpha$ from $B \rightarrow \rho \rho, \rho \pi, \pi \pi$ is only affected by NP in electroweak penguins [31]). The improvements in $\gamma$ beyond Phase I, shown in Table I, assume the so-called modelindependent measurement, without charm factory input [32]. The fits include the constraints from the measurements of $A_{\mathrm{SL}}^{d, s}$ [20,23], but not their linear combination [33] nor $\Delta \Gamma_{s}$, whose effects on the future constraints on NP studied in this paper are small.

Initial investigations at the physics case of FCC-ee are gathered in Ref. [34], and provide the starting ground of the present study. The inputs in Table I correspond to the actual sensitivity studies performed so far, which are only a subset of the observables to be improved. Most inputs considered in this work for Phase III are obtained from extrapolations (scaling to luminosity) of the current precision or sensitivity of the measurements obtained at Belle II and LHCb [34]. Some comments are, however, in order for two of them: $\left|V_{c b}\right|$ and $A_{\mathrm{SL}}$. The $\left|V_{c b}\right|$ sensitivity is derived from the counting of the $W$ decays selected with two jets satisfying $b$-tagging and $c$-tagging algorithms, which performance is given in Refs. [35,36]. It is already observed from this stateof-the-art starting point that the precision on the $\left|V_{c b}\right|$ matrix element is improved by a factor 3-4. The precision of the semileptonic $C P$ asymmetries are obtained from a fast simulation study [37]. A similar method as employed by LHCb [38] is considered, using a squared-cut based selection of the decays $B_{s} \rightarrow D_{s} \ell \nu X$, but enhanced to decays of $D_{s}$ containing $\pi^{0}$ and $K_{S}$. The obtained statistical precision is a few times $10^{-5}$, which makes possible to attain the SM value. However, the detection asymmetries are expected to be a limitation of the method, at a level comparable to the statistical uncertainty.

\section{B. Current status}

The present constraints on the magnitudes of NP contributions to the $B_{d}$ and $B_{s}$ mixing amplitudes are shown in Fig. 1, with inputs corresponding to the summer 2019 version of the CKMfitter Collaboration updates [28], to which we add the inputs $A_{\mathrm{SL}}^{d}=0.0000 \pm 0.0019$ and $A_{\mathrm{SL}}^{s}=+0.0016 \pm 0.0030$ (with $+6.6 \%$ correlation) [17]. In the SM fit $\left(h_{d}=h_{s}=0\right)$ the pulls of the observables $\Delta m_{d}$ and $\Delta m_{s}$ are 1.7 and $1.3 \sigma$, respectively. Allowing for

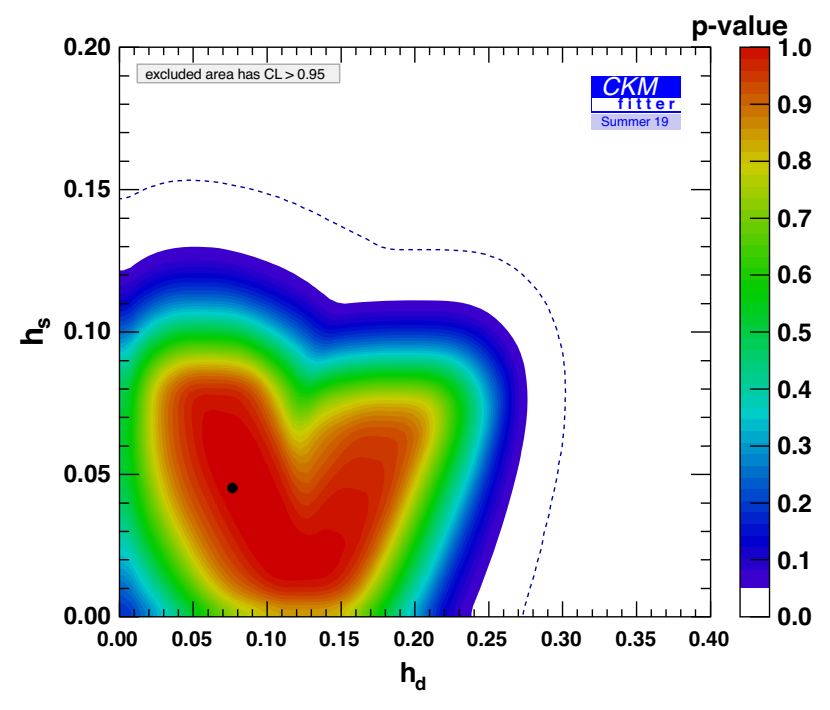

FIG. 1. Current sensitivities to $h_{d}-h_{s}$ in $B_{d}$ and $B_{s}$ mixings as of summer 2019 [28]. The black dot indicates the best-fit point, and the dotted curve shows the $99.7 \%$ CL $(3 \sigma)$ contour.

NP contributions, the fit shown in Fig. 1 favors $h_{d}$ and $h_{s}$ somewhat away from the origin, alleviating the pulls of $\Delta m_{d}$ and $\Delta m_{s}$ to 0.4 and $0.2 \sigma$, respectively. Figure 1 shows agreement with the SM hypothesis at $\sim 1 \sigma$.

In the NP scenario, the $1 \sigma$ intervals for the Wolfenstein parameters are

$$
\begin{aligned}
A & =0.813_{-0.015}^{+0.016}, & \lambda & =0.224835_{-0.000059}^{+0.000255}, \\
\bar{\rho} & =0.122_{-0.022}^{+0.025}, & \bar{\eta} & =0.371_{-0.015}^{+0.022} .
\end{aligned}
$$

Note that the uncertainties of $\bar{\rho}$ and $\bar{\eta}$ increase by about a factor of 3 compared to the fits assuming the SM, while for the NP parameters we obtain

$$
\begin{aligned}
h_{d} & =0.075_{-0.064}^{+0.153}, \quad h_{s}=0.048_{-0.048}^{+0.048}, \\
\sigma_{d} & =-1.40_{-0.23}^{+0.97},
\end{aligned}
$$

with $\sigma_{s}$ unconstrained at $1 \sigma$. The plot in Fig. 1 is obtained by treating $\bar{\rho}, \bar{\eta}$, and the other physics parameters not shown as nuisance parameters. This corresponds to the case of generic NP, ignoring possible model-dependent relations between different $\Delta F=2$ transitions. The constraint from $\epsilon_{K}$ has negligible impact throughout this paper when no NP in the kaon sector is considered; when NP in this sector is allowed as mentioned in the Introduction, $\epsilon_{K}$ probes NP mediating $\Delta S=2$ transitions, with no impact whatsoever on our analyses. One can see from Fig. 1 that $\mathrm{LHCb}$ measurements have imposed comparable constraints on NP in $B_{s}$ mixing to those in the $B_{d}$ system. This qualitative picture will continue to hold in the future.

To estimate and plot future sensitivities for our Phase I, II, and III benchmarks, we adjusted the central values of the 

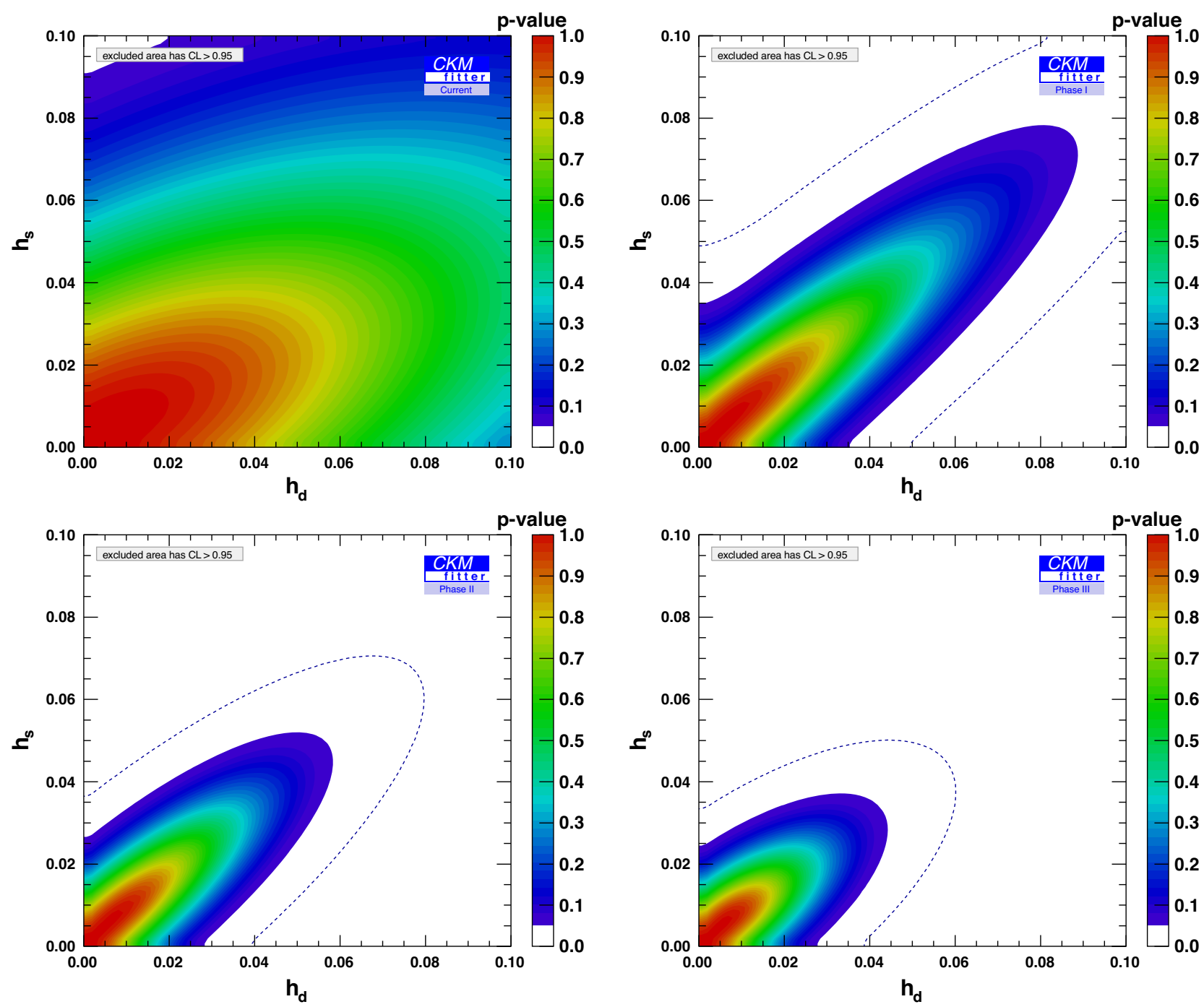

FIG. 2. Current (top left), Phase I (top right), Phase II (bottom left), and Phase III (bottom right) sensitivities to $h_{d}-h_{s}$ in $B_{d}$ and $B_{s}$ mixings, resulting from the data shown in Table I (where central values for the different inputs have been adjusted). The dotted curves show the $99.7 \%$ CL $(3 \sigma)$ contours.

input measurements to their best fit values in the SM global fit of 2019, in order to eliminate tensions when moving to smaller uncertainties in the future scenarios. The effect of adjusting the central values is illustrated by the top left plot in Fig. 2, which shows the fit with the adjusted central values of Table I and the same uncertainties as in Fig. 1. By construction, the $p$-value in Fig. 2 is maximal at $h_{d}=h_{s}=0$. It turns out that both fits yield similar $3 \sigma$ bounds on $h_{d}$ and $h_{s}$.

\section{Phase I exploration}

As indicated in Table I, compared to the current status, the uncertainties of many nonperturbative theoretical inputs are anticipated to be improved by a factor of at least 1.5 , up to 4 . In particular, uncertainties of the bag parameters and decay constants, necessary for predicting the mass differences of the two $B_{d}$ and $B_{s}$ mass eigenstates, will all go below the percent level. At the same time, Belle II will improve the determinations of the CKM matrix elements $\left|V_{c b}\right|$ and $\left|V_{u b}\right|$, by measuring the semileptonic channels $B \rightarrow D^{(*)} \ell \bar{\nu}$, and $B \rightarrow \pi \ell \bar{\nu}$. The LHCb collaboration has measured $\left|V_{c b}\right|$ for the first time at a hadronic machine [39] and is expected to contribute to the final precision of the world average. Yet, this is not taken into account in the anticipated precision of this observable considered here. Moreover, the uncertainties in the determinations of the angles of the $B_{d}$ unitary triangle will reach around the $1^{\circ}$ level.

These improvements on theoretical inputs and data translate into much better constraints on the $h_{d}-h_{s}$ plane parametrizing the size of NP in $B_{s}$ and $B_{d}$ meson-mixing, as seen from the top right plot in Fig. 2, which assumes that future measurements remain consistent with the SM. These results are similar to the "Stage II" scenario shown in 
TABLE II. The role of input uncertainties in the Phase I results, for LHCb with 50/fb and Belle II with 50/ab. The displayed $h_{d, s}$ ranges are at $1 \sigma$, and percentages correspond to the relative uncertainty with respect to the main fit.

\begin{tabular}{lll}
\hline \hline & \multicolumn{2}{c}{ Sensitivities at $1 \sigma$} \\
\cline { 2 - 3 } Fit description (Phase I) & \multicolumn{1}{c}{$h_{d}$} & $h_{s}$ \\
\hline Main fit & {$[0,0.040](100 \%)$} & {$[0,0.036](100 \%)$} \\
No $\left\{f_{B_{s}}, f_{B_{s}} / f_{B_{d}}, B_{B_{s}}, B_{B_{s}} / B_{B_{d}}\right\}$ uncertainties & {$[0,0.036](90 \%)$} & {$[0,0.033](92 \%)$} \\
No $\eta_{B}$ uncertainty & {$[0,0.035](88 \%)$} & {$[0,0.031](86 \%)$} \\
No $\left\{f_{B_{s}}, f_{B_{s}} / f_{B_{d}}, B_{B_{s}}, B_{B_{s}} / B_{B_{d}}, \eta_{B}\right\}$ uncertainties & {$[0,0.032](80 \%)$} & {$[0,0.029](81 \%)$} \\
\hline \hline
\end{tabular}

Ref. [12], which corresponded to the same projected LHCb and Belle II integrated luminosities.

Table II illustrates the effects of reducing the uncertainties of the nonperturbative and perturbative theoretical inputs involved in the predictions of the mass differences $\Delta m_{d}$ and $\Delta m_{s}$, where we explored the consequences of eliminating their uncertainties. This table shows that setting to zero the uncertainties of the nonperturbative or the perturbative theoretical inputs have similar impacts on the allowed ranges of the NP parameters $h_{d}$ and $h_{s}$, with an improvement of about $10 \%$ for each.

\section{Phase II exploration}

We now shift to the sensitivity to NP achievable in Phase II, shown in the bottom left plot in Fig. 2. As seen from Table I, some key quantities such as $\phi_{s}, \gamma$ or $\beta$ will be much more precisely determined (typically by a factor of 3 ). Yet some other key quantities will only be slightly improved. This is the case for the bag parameters, and also for the uncertainties in the extractions of $\left|V_{c b}\right|$ and $\left|V_{u b}\right|$ from semileptonic decays. The reliability of these extrapolations (pertinent for the late 2030s) is necessarily less good than for Phase I (i.e., late 2020s).

As shown in Fig. 2, the constraints on $h_{d}$ and $h_{s}$ will improve again between Phase I and Phase II, although the improvement is smaller than that between the summer 2019 situation and Phase I. This is caused only in part by the slight pull away from the SM seen in the summer 2019 fit in Fig. 1.

To understand the future limitations, we compare in Table III the impact on the sensitivity to $h_{d, s}$ when improving or eliminating the uncertainties of some key quantities in the computations of $\Delta m_{d}$ and $\Delta m_{s}$. As this table shows, $\left|V_{c b}\right|$ plays a central role: neglecting its uncertainty (implemented by a factor of 20 reduction), sensitivities improve by about $25 \%$, and the improvements increase up to $70 \%$ when eliminating simultaneously the uncertainties coming from perturbative and nonperturbative theoretical inputs for meson mixing, as illustrated in Fig. 3. Recall that a precise determination of $\left|V_{c b}\right|$ amounts to a precise determination of the Wolfenstein parameter $A$. Therefore, our findings imply that the remaining Wolfenstein parameters $\bar{\rho}$ and $\bar{\eta}$ are precise enough at Phase II, while the determination of $A$ is a bottleneck for constraining further $h_{d}$ and $h_{s}$. The effect of improving only the theoretical inputs is also shown in Table III, where an improvement in sensitivity by roughly $25 \%$ is seen when eliminating the uncertainties of all theoretical inputs for meson mixing.

We should emphasize that many other measurements will improve substantially in Phase II, e.g., the uncertainties of $\mathcal{B}\left(B_{s} \rightarrow \mu \mu\right)$ and $\mathcal{B}\left(B_{d} \rightarrow \mu \mu\right)$ are expected to reach $5 \%$ and $10 \%$, respectively [30]. They will also provide high sensitivity to (other types of) NP, but do not impact the constraints on $h_{d, s}$ and $\sigma_{d, s}$.

TABLE III. The role of input uncertainties in Phase II results, for LHCb with 300/fb and Belle II with 250/ab. We analyze the impact on bounds for $h_{d}$ and $h_{s}$ when: (a) we reduce by a factor of 20 the uncertainty of various key quantities for calculating $\Delta m_{d}$ and $\Delta m_{s}$ and (b) the $\left\{f_{B_{s}}, f_{B_{s}} / f_{B_{d}}, B_{B_{s}}, B_{B_{s}} / B_{B_{d}}, \eta_{B}\right\}$ uncertainties are set to zero (also denoted as "no theor. uncert."). Percentages correspond to the relative uncertainty with respect to the main fit.

\begin{tabular}{|c|c|c|c|}
\hline \multirow[b]{2}{*}{ Fit description (Phase II) } & \multicolumn{2}{|c|}{ Sensitivities at $1 \sigma$} & \multirow[b]{2}{*}{ Plot in Fig. 3} \\
\hline & $h_{d}$ & $h_{s}$ & \\
\hline Main fit & {$[0,0.028](100 \%)$} & {$[0,0.025](100 \%)$} & Top left \\
\hline No $\left\{f_{B_{s}}, f_{B_{s}} / f_{B_{d}}, B_{B_{s}}, B_{B_{s}} / B_{B_{d}}\right\}$ uncertainties & {$[0,0.024](86 \%)$} & {$[0,0.023](92 \%)$} & $\ldots$ \\
\hline No $\eta_{B}$ uncertainty & {$[0,0.024](86 \%)$} & {$[0,0.021](84 \%)$} & $\ldots$ \\
\hline No $\left\{f_{B_{s}}, f_{B_{s}} / f_{B_{d}}, B_{B_{s}}, B_{B_{s}} / B_{B_{d}}, \eta_{B}\right\}$ uncertainties & {$[0,0.020](71 \%)$} & {$[0,0.019](76 \%)$} & Top right \\
\hline$\delta_{\mathrm{tot}}\left|V_{c b}\right|_{\mathrm{SL}} / 20$ & {$[0,0.022](79 \%)$} & {$[0,0.018](72 \%)$} & Bottom left \\
\hline$\delta_{\mathrm{tot}}\left|V_{c b}\right|_{\mathrm{SL}} / 20$, "no theor. uncert." & {$[0,0.0096](34 \%)$} & {$[0,0.0061](24 \%)$} & Bottom right \\
\hline$\left\{\delta_{\text {tot }}\left|V_{u b}\right|_{\mathrm{SL}}, \delta_{\mathrm{tot}}\left|V_{c b}\right|_{\mathrm{SL}}, \delta \sin (2 \beta), \delta \sin (2 \gamma)\right\} / 20$, "no theor. uncert." & {$[0,0.0089](32 \%)$} & {$[0,0.0061](24 \%)$} & $\ldots$ \\
\hline
\end{tabular}



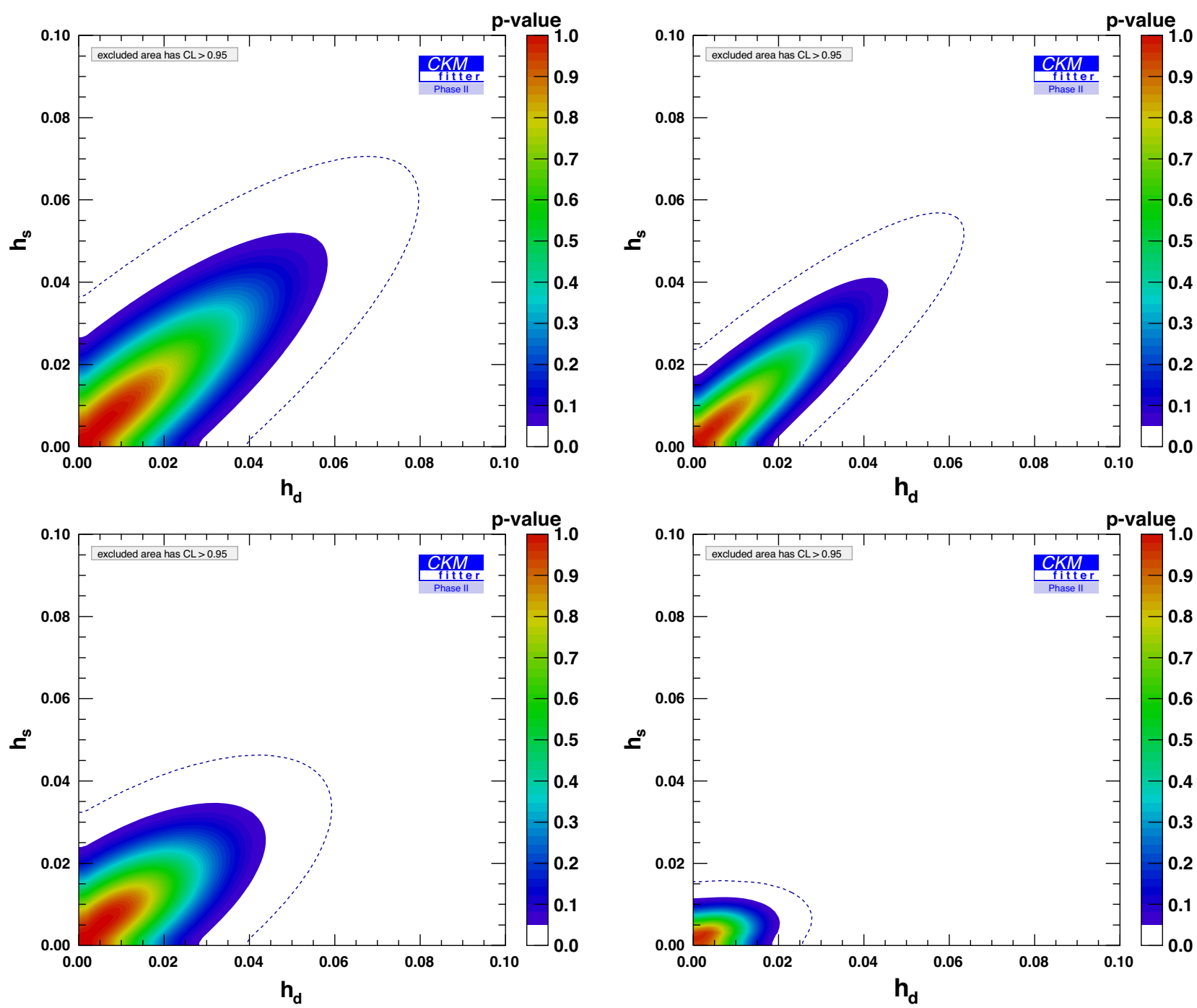

FIG. 3. Impact of improving key uncertainties in Phase II: nominal Phase II plot (top left, same as bottom left in Fig. 2), case in which uncertainties from the perturbative and nonperturbative theoretical inputs $\left\{f_{B_{s}}, f_{B_{s}} / f_{B_{d}}, B_{B_{s}}, B_{B_{s}} / B_{B_{d}}, \eta_{B}\right\}$ are eliminated (top right), case in which the extraction of $\delta_{\text {tot }}\left|V_{c b}\right|_{\mathrm{SL}}$ is improved by a factor 20 (bottom left, note the similarity with Phase III in Fig. 2), combining improvements on theoretical inputs and $\delta_{\text {tot }}\left|V_{c b}\right|_{\mathrm{SL}}$ (bottom right).

Note also the importance of lattice QCD uncertainties. Their predicted improvements are more uncertain the more one extrapolates into the future. Two sets of recent predictions for the decay constants and bag parameters are shown in Table IV. Our results in Fig. 2 are based

TABLE IV. Predictions for future lattice QCD uncertainties.

\begin{tabular}{llllll}
\hline \hline & \multicolumn{2}{c}{ Ref [30] (LHCb) } & & Ref. [29] (Belle II 10 yrs) \\
\cline { 2 - 3 } \cline { 5 - 6 } & 2025 & 2035 & & & \\
Uncertainties & $(23 / \mathrm{fb})$ & $(300 / \mathrm{fb})$ & & "w/EM" & “w/o EM” \\
\hline$\delta f_{B_{s}}$ & 0.0011 & 0.0011 & & 0.0024 & 0.00074 \\
$\delta\left(f_{B_{s}} / f_{B_{d}}\right)$ & 0.005 & 0.005 & & 0.012 & 0.0014 \\
$\delta B_{B_{s}}$ & 0.010 & 0.007 & & 0.018 & 0.012 \\
$\delta\left(B_{B_{s}} / B_{B_{d}}\right)$ & 0.005 & 0.003 & & 0.013 & 0.0072 \\
\hline \hline
\end{tabular}

on Ref. [30] for these inputs. Using instead the "10 yrs w/o EM" values from Ref. [29] for Phase II (assuming that electromagnetic effects will be included on the Phase II timescale) would yield very similar results, since form factor projections are more optimistic, while the opposite holds for the bag parameters. The combination of Refs. [29,30] (i.e., considering the most optimistic projections in Table IV) leads to a slight improvement in the sensitivities to $h_{d}$ and $h_{s}$, and a strong improvement in their correlation, due to the significantly smaller uncertainty of $f_{B_{s}} / f_{B_{d}}$ with respect to [30].

\section{E. Phase III exploration}

The sensitivity achievable in Phase III is displayed in the bottom right plot in Fig. 2. No improvement in lattice QCD uncertainties is used in going from Phase II to Phase III, 
TABLE V. Current and future 95\% CL sensitivities to $h_{d, s}$, assuming unrelated NP contributions in $B_{d, s}$ mixings.

\begin{tabular}{ccccc}
\hline \hline Sensitivities & Summer 2019 & Phase I & Phase II & Phase III \\
\hline$h_{d}$ & 0.26 & 0.073 & 0.049 & 0.038 \\
$h_{s}$ & 0.12 & 0.065 & 0.044 & 0.031 \\
\hline \hline
\end{tabular}

since we are not aware of any predictions for the relevant time frame. Hence, these sensitivity projections are probably (very) conservative. The observed improvement in sensitivity to $h_{d}$ and $h_{s}$ from Phase II to Phase III is therefore solely related to the improvement in $\left|V_{c b}\right|$ precision at FCC-ee. The projections provided at Phase II and even more at Phase III support the need for simultaneously improving the CKM normalization and the hadronic parameters describing the mixing, to fully exploit the precision of the CKM observables at these time frames. Any improvements beyond what can currently be anticipated would make the Phase III sensitivity better than plotted in Fig. 2. It should also be emphasized that the FCC-ee program has a much broader scope than the study discussed in this paper.

\section{INTERPRETATIONS}

The 95\% CL sensitivities to $h_{d}$ and $h_{s}$ obtained above are summarized in Table V. The energy scales probed by meson mixing can be doubled due to the anticipated improvement in the sensitivity to $h_{d}$, going from the current constraints to Phase I (improvement by more than a factor of 3) and to Phase II (factor of 5). These improvements compare well with those anticipated in the NP reach of the HL-LHC, during the same time frame.

The sensitivities to $h_{d, s}$ are straightforward to convert to the scales of BSM operators probed. While BSM models may generate (combinations of) several distinct dimension6 four-fermion operators contributing to $B-\bar{B}$ mixing, for illustration we only calculate here the sensitivities to the scales of the operators which occur in the SM, shown in Eq. (2). We use Eq. (3) and distinguish several scenarios. For NP with flavor structure independent of the SM Yukawa couplings, we set $\left|C_{i j}\right|$ to unity. Many NP models contain suppressions of flavor-changing processes similar to the SM, in which case $\left|C_{i j}\right|=\left|\lambda_{i j}^{t}\right|$ may be appropriate (we use Ref. [28] for the numerical values of $\left|\lambda_{i j}^{t}\right|$ ). For NP contributions that occur at tree level, the $(4 \pi)^{2}$ factor in Eq. (3) is present, while it should be removed if the NP contribution is generated at the one-loop level (similar to the SM box diagrams).

The resulting sensitivities to NP energy scales are shown in Table VI up to Phase II. The scales probed at Phase III are not shown, since they are primarily dependent on not yet estimated lattice QCD improvements at this time frame. Nevertheless, we note that FCC-ee precision measurements would improve significantly the mixing analyses if the bottlenecks that we identified $\left(\left|V_{c b}\right|\right.$ and lattice QCD parameters) can be addressed.

One sees that even if NP contains the same CKM suppressions of $\Delta F=2$ transitions as those present for the SM contributions, as well as a one-loop suppression, both of which occur for many NP models which are in the LHC energy range, the scales probed by the mixing constraints are still at the $1-2 \mathrm{TeV}$ range. These are comparable to gluino masses explored at the HL-LHC, and provide comparable sensitivity to NP as high- $p_{T}$ searches.

If the NP contributions to neutral meson mixing do not have either a loop suppression or CKM suppression (or neither), then the scale sensitivity is much higher, extending to thousands of TeV. It is indeed very easy to add NP to the SM, well outside the energy range of any current or future collider, which could still have an observable impact in flavor physics measurements.

So far in this paper we assumed that future measurements agree with the SM predictions. However, future data can not only set better bounds on NP, they may also reveal deviations from the SM. This is illustrated in Fig. 4, where we set the CKM parameters as well as $h_{d, s}$ and $\sigma_{d, s}$ to their current best fit values (allowing for NP in $\Delta F=2$; i.e., the point indicated by the black dot in Fig. 1), and performed a fit assuming for all future measurements the corresponding central values, but uncertainties as given in Table I for Phases I and II. While any assumption about possible future NP signals include a high degree of arbitrariness, Fig. 4 gives an impression of the sensitivity to reveal a deviation from the SM.

TABLE VI. The scale of the operator in Eq. (2) probed (in TeV, at 95\% CL) by $B_{d}$ and $B_{s}$ mixings at present, at Phase I, and Phase II, if the NP contributions in the two meson mixings are unrelated. The impact of SM-like hierarchy of couplings and/or loop suppression is shown.

\begin{tabular}{|c|c|c|c|c|c|c|c|}
\hline \multirow[b]{2}{*}{ Couplings } & \multirow[b]{2}{*}{ NP loop order } & \multicolumn{2}{|c|}{ Sensitivity for Summer $2019[\mathrm{TeV}]$} & \multicolumn{2}{|c|}{ Phase I Sensitivity [TeV] } & \multicolumn{2}{|c|}{ Phase II Sensitivity [TeV] } \\
\hline & & $B_{d}$ mixing & $B_{s}$ mixing & $B_{d}$ mixing & $B_{s}$ mixing & $B_{d}$ mixing & $B_{s}$ mixing \\
\hline$\left|C_{i j}\right|=\left|V_{t i} V_{t j}^{*}\right|$ & Tree level & 9 & 13 & 17 & 18 & 20 & 21 \\
\hline (CKM-like) & One loop & 0.7 & 1.0 & 1.3 & 1.4 & 1.6 & 1.7 \\
\hline$\left|C_{i j}\right|=1$ & Tree level & $1 \times 10^{3}$ & $3 \times 10^{2}$ & $2 \times 10^{3}$ & $4 \times 10^{2}$ & $2 \times 10^{3}$ & $5 \times 10^{2}$ \\
\hline (No hierarchy) & One loop & 80 & 20 & $2 \times 10^{2}$ & 30 & $2 \times 10^{2}$ & 40 \\
\hline
\end{tabular}



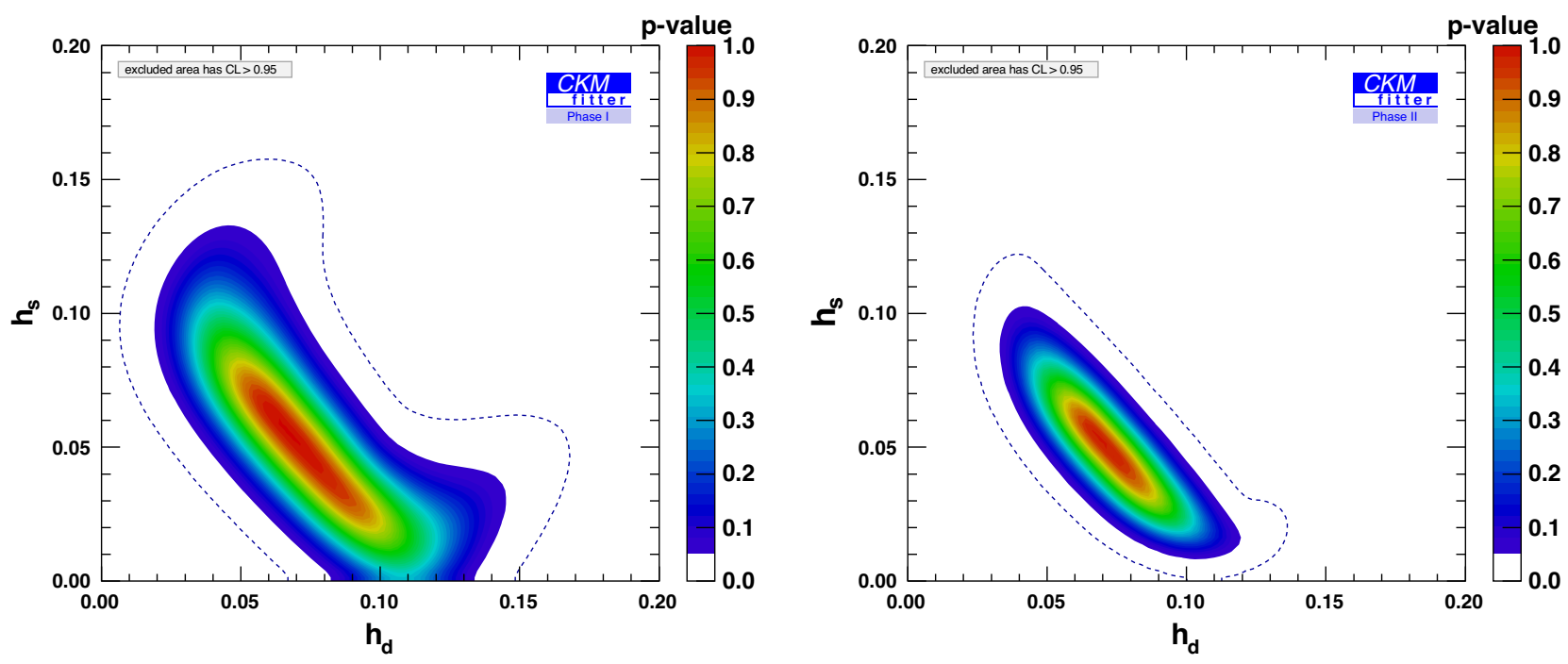

FIG. 4. Discovery prospects at Phase I (left) and Phase II (right), if the central values are as in the summer 2019 fit in Fig. 1.

\section{PERSPECTIVES AND LIMITATIONS ON $\left|V_{c b}\right|$ IMPROVEMENT}

For our analysis, precise determinations of CKM parameters from tree-level measurements is essential. This is particularly important when one reaches the Phase II precision, where we identified the $\left|V_{c b}\right|$ precision (together with the hadronic mixing parameters) as the bottleneck for this analysis. This section aims at sketching novel ways to measure the matrix element $\left|V_{c b}\right|$.

Currently there is an approximately $3 \sigma$ tension in the measurements of $B \rightarrow D^{(*)} \ell \nu$ semileptonic decays, when the rate to $\tau$ leptons is compared to the average of the $e, \mu$ modes [17,45]. Furthermore, the inclusive $\left|V_{c b}\right|$ and $\left|V_{u b}\right|$ measurements also differ by more than $3 \sigma$ from their exclusive counterparts [46], when considered together.

The tension between inclusive and exclusive $\left|V_{u b}\right|$ and $\left|V_{c b}\right|$ determinations might also remain a cause for concern [47]. If these discrepancies are not resolved and further established with higher significance in the coming years (by LHCb and Belle II), they would also impact the analysis of NP contributions to meson mixing.

\section{A. Opportunities for more precise $\left|\boldsymbol{V}_{\boldsymbol{c}}\right|$ determinations}

There are several possible ways to make progress in the future concerning $\left|V_{c b}\right|$. For the determination of $\left|V_{c b}\right|$ from exclusive semileptonic decays, separately measuring and computing the isospin difference between $B^{0}$ and $B^{ \pm}$ decays would allow to cross check the experimental analysis against lattice QCD, whose systematic uncertainties (after including electromagnetic corrections) can reach subpercent level. For the inclusive determination, similar isospin tests would be even more stringent, since isospin breaking effects are also suppressed by powers of $\Lambda_{\mathrm{QCD}} / m_{b}$, and should hence be negligible, with the only outstanding issues coming from isospin-breaking induced by QED radiative corrections, which require matching theoretical calculations with the specific setup of experimental analyses.

The direct determination of the CKM matrix element $\left|V_{c b}\right|$ at a high-luminosity $W$ factory (FCC-ee) has been used as an input for Phase III. Given the anticipated number of $W$ decays [34], the ultimate statistical precision that can be achieved is of $\mathcal{O}\left(10^{-4}\right)$ which corresponds to about two orders of magnitude improvement with respect to the current precision. The key ingredient for this kind of measurements is the capability of the $c$-and $b$-jet tagging algorithms to reject the lighter quark flavors.

Another opportunity for improvement can arise at a highluminosity $Z$-factory, such as FCC-ee, where $B_{c} \rightarrow \tau \nu$ decay can be reconstructed and a measurement of the product of the $B_{c}$ production fraction times the branching fraction of interest can be expected at the level of 1\% [48]. The challenge of the interpretation of the measurement stands in the knowledge of the $B_{c}$ production fraction at the $Z$ pole, where no input from $B$ factories exists. Its determination must rely on the theoretical prediction of exclusive decay branching fractions or their experimental measurement at an $e^{+} e^{-}$collider. The $B_{c}^{(*)}$ pair production cross section near threshold is of the order of $\mathcal{O}$ (few fb) [49] (dominated by the $V V$ and $V P$ channels), and therefore would require the collection of $\mathcal{O}(10 / \mathrm{ab})$ around $\sqrt{s} \sim 15 \mathrm{GeV}$.

\section{B. If NP contributes to semileptonic $B$ decay}

The discrepancies in semileptonic $B$ decay measurements may be due to currently underestimated theoretical or experimental uncertainties or could potentially be (at least partially) explained by the presence of BSM contributions in charged current processes. In particular, NP in the $\ell=\tau$ channel may yield violations of lepton flavor 
universality (LFU), while NP in the $\ell=e, \mu$ channels can both produce LFU deviations and potentially contribute to the inclusive vs exclusive tensions. The $\tau$ case has been extensively investigated due to the fact that BSM models explaining the $R\left(D^{(*)}\right)$ anomalies by modifying semitauonic processes are less constrained by other measurements [50-57]. On the other hand, BSM contributions in the $e, \mu$ channels have received less attention [58-63]. In particular, the question of the maximum size of the NPinduced deviations in these observables achievable in viable models that respect other experimental constraints has not been fully studied. This is relevant here, as it also corresponds to a violation of one of the assumptions of the analysis performed in this paper, namely that charged current processes are not significantly affected by BSM physics. Nevertheless, we now show that the $h_{d, s}-\sigma_{d, s}$ fit is still relevant for this particular scenario.

If the current anomalies in $b \rightarrow c \tau \bar{\nu}$ decays are attributed to BSM physics, that would imply that NP must exist at or below the TeV scale. Depending on the specific model that is UV-completing the dimension-6 operators, ATLAS and CMS should have a good chance to directly produce and detect the particle(s) mediating such charged current interactions. On the other hand, in some of these models, direct high- $p_{T}$ searches may not fully exclude BSM contributions at a level that they could still affect $\left|V_{c b}\right|$ and $\left|V_{u b}\right|$ measurements at a precision attainable in Phases I-III.

In this case, complementary flavor physics observables can provide further insights. For example, if the NP contributions to $b \rightarrow c \ell \nu$ transitions has a different Lorentz structure than the SM, it will manifest itself by modifying kinematic properties of the decays, e.g., the charged lepton energy or $q^{2}$ spectrum, the $\tau$ polarization, etc. Such NP effects may be therefore disentangled from the SM pure $V-A$ contributions, and one could in principle perform a combined fit and extract $\left|V_{c b}\right|$ while constraining NP in semileptonic transitions. In such case the future precision to which $\left|V_{c b}\right|$ (and similarly $\left|V_{u b}\right|$ ) will be known, is going to be likely worse than assumed in Table I. Further quantitative studies are needed to assess how much the projections performed here will be degraded and are beyond the scope of this work.

On the other hand, if NP generates the same $V-A$ interaction as the SM (or if the contribution is smaller than what kinematic distributions can constrain), it will bias the measurement of $\left|V_{c b}\right|$ and $\left|V_{u b}\right|$. In this case such tree-level NP effects will be seen in this analysis as nonzero contributions to $h_{d, s}$ and $\sigma_{d, s}$, in the neutral kaon system, and in many $\Delta F=1$ processes [63]. These deviations from the SM induced by corrections to the charged currents will be present on top of genuine BSM FCNC contributions, which modifies the interpretation of the quantities extracted once NP is allowed and may require one to reorganise the fit of the CKM parameters as a consequence [64].
The fit performed here assumes the unitarity of the SM and that charged currents are only produced by SM processes. Therefore the experimental determination of, e.g., $V_{c b}^{\mathrm{exp}}=V_{c b}^{\mathrm{SM}}+\delta V_{c b}$ is used by the fit to determine via unitarity the CKM combinations entering meson mixing, such as $\left(V_{t b} V_{t s}^{*}\right)^{\text {fit }}$ which are then compared with their experimental counterparts $\left(V_{t b} V_{t s}^{*}\right)$ exp, with the discrepancies being attributed to $\Delta F=2 \mathrm{NP}$ contributions via the $h$ and $\sigma$ parameters. Similar redefinitions hold for other CKM entries determined in charged current processes, such as $\left|V_{u b}\right|$ (and to the entries in the first two generations CKM submatrix, although their impact is less appreciable due to the better precision to which they are known). This remains true as long as the tree level determination of products of $V_{t s}, V_{t d}$, and $V_{t b}$ is not reaching the precision attainable from FCNC processes, and therefore is inferred from unitarity. This situation will hold in the foreseeable future. So, while in the introduction we have simplified the presentation by assuming that the tree-level processes are unchanged by new physics, $h_{s, d}$ and $\sigma_{s, d}$ really parameterize generic "tree vs loop" type discrepancies.

More concretely, assuming that NP pollutes $V_{c b}$ at tree level by $\delta V_{c b}$ and similarly for $V_{u b}, V_{t s}, V_{t d}$ (while neglecting contributions to $V_{t b}, V_{c s}, V_{c d}, V_{u s}, V_{u d}$ for clarity), at leading order in both $m_{c} / m_{t}$ and in the size of new physics, $v^{2} / \Lambda^{2}$, we have:

$h_{d} e^{2 i \sigma_{d}} \simeq 2\left(V_{t b}^{*} \delta V_{t d}+\delta V_{c b}^{*} V_{c d}+\delta V_{u b}^{*} V_{u d}\right) /\left(V_{t b}^{*} V_{t d}\right)$,

$h_{s} e^{2 i \sigma_{s}} \simeq 2\left(V_{t b}^{*} \delta V_{t s}+\delta V_{c b}^{*} V_{c s}+\delta V_{u b}^{*} V_{u s}\right) /\left(V_{t b}^{*} V_{t s}\right)$,

which should be added to the genuine NP contributions in mixing. The full (nonlinear) expressions can also be straightforwardly derived. Notice that the presence of some $\delta V_{i j}$ do not necessarily imply the presence of others for different families $i, j$, since some of these contributions may arise from operators involving right-handed quarks (below the level that can be constrained by kinematic distributions) which are unrelated by $S U(2)_{L}$ symmetry.

The same tree-level induced deviations from the SM predictions will appear also in all $\Delta F=1$ processes, of similar size at the level of the branching ratios to the contribution to $\Delta F=2$ processes. This can be used to characterize the NP contributions and potentially disentangle the effects coming from measurements in charged-current processes via unitarity from genuine loop contributions. Furthermore, since the precision with which most of the $\Delta F=1$ decays will be able to constrain NP is unlikely to reach a similar level of accuracy with which $h_{s, d}$ will be constrained in Phase III, there exists scenarios where the $\Delta F=2 \mathrm{NP}$ fit may still be one of the first places where NP affecting flavor changing charged currents will show up. We leave the identification of suitable example models to future work.

Notice also that, in the language of the SMEFT, semileptonic $B$ decays are affected by both four-fermion 
operators and operators involving a (flavor violating) quark bilinear, covariant derivatives and Higgs fields, as such operators directly modify the $W b c$ vertex at order $v^{2} / \Lambda^{2}$. Therefore, the above discussion applies to the combined effect of all such operators during Phase I and II, as the most precise determinations of $\left|V_{c b}\right|$ are at low energy. On the other hand, at Phase III, $\left|V_{c b}\right|$ will also be well measured via $W$ decays at FCC-ee. Such measurement will not be affected by four-fermion operators and the operators whose effects cannot be disentangled in the $\left|V_{c b}\right|$ measurements will only be those modifying the $W b c$ vertex.

\section{SUMMARY AND OUTLOOK}

The constraints on new physics in $B_{d}$ and $B_{s}$ mixings have been determined in light of recent measurements, in particular from the $\mathrm{LHCb}$ experiment and the $B$ factories. These results update those published in Refs. [22,28]. A good agreement with the SM is obtained, with an increased precision compared to our earlier results. As shown in Fig. 1, up to $\sim 20 \%$ NP contributions to the mixing amplitudes are still allowed, relative to the SM contributions, and press to consider the prospects at future facilities.

The long-term experimental prospects for flavor physics involve now three proposals, with different timelines and maturity: the LHCb Upgrade II at the high luminosity LHC, the recently initiated possible Belle II upgrade, and finally the FCC-ee machine including a high-luminosity $Z$-factory phase to succeed the HL-LHC program at CERN. We found that if no NP signal is seen, the bounds on $h_{d}$ and $h_{s}$ will improve by about a factor of 3 after the first $\mathrm{LHCb}$ upgrade and the Belle II completions (Phase I), in line with the results obtained in Ref. [12], confirming the impressive progress expected from the LHCb upgrade and the Belle II experiment.

Though steady improvements in precision of the main observables are achieved at each of the benchmark phases considered, they do not fully reflect into the $h_{d}$ and $h_{s}$ sensitivities. We identified these bottlenecks in precision to the determination of both $\left|V_{c b}\right|$ and the hadronic parameters of neutral-meson mixing. In relation with this question, and motivated by the tension with lepton flavor universality in $B \rightarrow D\left(^{*}\right) \ell \nu$, we discussed how the future facilities could improve on $\left|V_{c b}\right|$, and how it would be affected by NP in semileptonic $B$ decays. Mixing observables have historically been a place of essential discoveries establishing the standard model, and provided crucial constraints on new physics model building. They will continue to play similar fundamental roles in the future.

\section{ACKNOWLEDGMENTS}

We thank Y. Grossman and A. Poluektov for helpful conversations on $\gamma$. We would like to thank all members of the CKMfitter group for suggestions on various aspects of this paper. Z. L. was supported in part by the Office of High Energy Physics of the U.S. Department of Energy under contract No. DE-AC02-05CH11231. M. P. is grateful for the support provided by the Walter Burke Institute for Theoretical Physics. L. V. S. was supported in part by the Spanish Government and ERDF funds from the EU Commission [Grant FPA2017-84445-P] and the Generalitat Valenciana [Grant Prometeo/2017/053].
[1] M. Kobayashi and T. Maskawa, Prog. Theor. Phys. 49, 652 (1973).

[2] J. Charles, A. Hocker, H. Lacker, S. Laplace, F. R. Le Diberder, J. Malcles, J. Ocariz, M. Pivk, and L. Roos (CKMfitter Group), Eur. Phys. J. C 41, 1 (2005).

[3] Z. Ligeti, Int. J. Mod. Phys. A 20, 5105 (2005).

[4] J. M. Soares and L. Wolfenstein, Phys. Rev. D 47, 1021 (1993).

[5] T. Goto, N. Kitazawa, Y. Okada, and M. Tanaka, Phys. Rev. D 53, 6662 (1996).

[6] J. P. Silva and L. Wolfenstein, Phys. Rev. D 55, 5331 (1997).

[7] Y. Grossman, Y. Nir, and M. P. Worah, Phys. Lett. B 407, 307 (1997).

[8] A. Hocker and Z. Ligeti, Annu. Rev. Nucl. Part. Sci. 56, 501 (2006).

[9] A. Hocker, H. Lacker, S. Laplace, and F. Le Diberder, Eur. Phys. J. C 21, 225 (2001).

[10] E. E. Jenkins, A. V. Manohar, and P. Stoffer, J. High Energy Phys. 03 (2018) 016.
[11] M. Endo, T. Kitahara, and D. Ueda, J. High Energy Phys. 07 (2019) 182.

[12] J. Charles, S. Descotes-Genon, Z. Ligeti, S. Monteil, M. Papucci, and K. Trabelsi, Phys. Rev. D 89, 033016 (2014).

[13] A. J. Buras, S. Jager, and J. Urban, Nucl. Phys. B605, 600 (2001).

[14] R. Aaij et al. (LHCb Collaboration), arXiv:1808.08865.

[15] Belle II VXD open workshop on possible future technologies for a collider with $4 \times 10^{36} \mathrm{~cm}^{-2} \mathrm{~s}^{-1}$ luminosity, https://indico.cern.ch/event/810687/ (2019), CERN.

[16] A. Blondel and F. Zimmermann, arXiv:1112.2518.

[17] Y.S. Amhis et al. (HFLAV Collaboration), arXiv:1909 .12524

[18] J. Charles et al., Phys. Rev. D 84, 033005 (2011).

[19] S. Descotes-Genon and P. Koppenburg, Annu. Rev. Nucl. Part. Sci. 67, 97 (2017).

[20] A. Lenz, U. Nierste, J. Charles, S. Descotes-Genon, A. Jantsch, C. Kaufhold, H. Lacker, S. Monteil, V. Niess, and S. T'Jampens, Phys. Rev. D 83, 036004 (2011). 
[21] A. Lenz, U. Nierste, J. Charles, S. Descotes-Genon, H. Lacker, S. Monteil, V. Niess, and S. T'Jampens, Phys. Rev. D 86, 033008 (2012).

[22] J. Charles et al., Phys. Rev. D 91, 073007 (2015).

[23] S. Laplace, Z. Ligeti, Y. Nir, and G. Perez, Phys. Rev. D 65, 094040 (2002).

[24] K. Agashe, M. Papucci, G. Perez, and D. Pirjol, arXiv: hep-ph/0509117.

[25] Z. Ligeti, M. Papucci, and G. Perez, Phys. Rev. Lett. 97, 101801 (2006).

[26] G. Isidori, Y. Nir, and G. Perez, Annu. Rev. Nucl. Part. Sci. 60, 355 (2010).

[27] Z. Ligeti, M. Papucci, G. Perez, and J. Zupan, Phys. Rev. Lett. 105, 131601 (2010).

[28] Summer 2019 CKMfitter updates, http://ckmfitter.in2p3.fr/.

[29] W. Altmannshofer et al. (Belle II Collaboration), Prog. Theor. Exp. Phys. (2019), 123C01; (2020), 029201(E).

[30] A. Cerri et al., CERN Yellow Rep. Monogr. 7, 867 (2019).

[31] J. Charles, O. Deschamps, S. Descotes-Genon, and V. Niess, Eur. Phys. J. C 77, 574 (2017).

[32] A. Poluektov, in Proceedings at the workshop towards the ultimate precision in flavour physics, University of Warwick, 2018, https://indico.cern.ch/event/694666/contributions/ 2916502/attachments/1635557/2609390/Poluektov_TUPFP .pdf.

[33] V. M. Abazov et al. (DØ Collaboration), Phys. Rev. D 84, 052007 (2011).

[34] A. Abada et al. (FCC Collaboration), Eur. Phys. J. C 79, 474 (2019).

[35] T. Behnke, J. E. Brau, B. Foster, J. Fuster, M. Harrison, J. M. Paterson, M. Peskin, M. Stanitzki, N. Walker, and H. Yamamoto, arXiv:1306.6327.

[36] M. H. Schune, in Proceedings at the 3rd FCC Physics and Experiments Workshop, CERN, 2020, https://indico .cern.ch/event/838435/contributions/3635812/attachments/ 1971221/3279502/FCCee_17Jan2020_v2.pdf (2020).

[37] S. Monteil, in Proceedings at the 4th FCC Week, Brussels, 2019, https://indico.cern.ch/event/727555/contributions/ 3467537/attachments/1870013/3076707/FCC_Brussels_ Flavours_monteil.pdf.

[38] R. Aaij et al. (LHCb Collaboration), Phys. Rev. Lett. 117, 061803 (2016); 118, 129903(A) (2017).

[39] R. Aaij et al. (LHCb Collaboration), Phys. Rev. D 101, 072004 (2020).

[40] Private communication from H. Li and H. Ma in preparation for the White Paper on BES-III Experiment.

[41] M. Ablikim et al., Chin. Phys. C 44, 040001 (2020).
[42] N. Carrasco et al. (ETM Collaboration), J. High Energy Phys. 03 (2014) 016.

[43] A. Bazavov et al. (Fermilab Lattice and MILC Collaborations), Phys. Rev. D 93, 113016 (2016).

[44] G. Buchalla, A. J. Buras, and M. E. Lautenbacher, Rev. Mod. Phys. 68, 1125 (1996).

[45] S. Bifani, S. Descotes-Genon, A. Romero Vidal, and M.-H. Schune, J. Phys. G 46, 023001 (2019).

[46] S. Aoki et al. (Flavour Lattice Averaging Group), Eur. Phys. J. C 80, 113 (2020).

[47] M. Tanabashi et al. (Particle Data Group Collaboration), Phys. Rev. D 98, 030001 (2018).

[48] S. Prell, https://indico.ihep.ac.cn/event/9960/session/12/ contribution/95/material/slides/0.pdf, Proceedings at the 2019 International Workshop on the High Energy Circular Electron Positron Collider, IHEP, Beijing, https://indico .ihep.ac.cn/event/9960/ (2019).

[49] A. Berezhnoy, A. Likhoded, A. Onishchenko, and S. Poslavsky, Nucl. Phys. B915, 224 (2017).

[50] D. Buttazzo, A. Greljo, G. Isidori, and D. Marzocca, J. High Energy Phys. 11 (2017) 044.

[51] A. Azatov, D. Bardhan, D. Ghosh, F. Sgarlata, and E. Venturini, J. High Energy Phys. 11 (2018) 187.

[52] C. Murgui, A. Penuelas, M. Jung, and A. Pich, J. High Energy Phys. 09 (2019) 103.

[53] D. Bardhan and D. Ghosh, Phys. Rev. D 100, 011701 (2019).

[54] P. Asadi and D. Shih, Phys. Rev. D 100, 115013 (2019).

[55] J. Aebischer, J. Kumar, P. Stangl, and D. M. Straub, Eur. Phys. J. C 79, 509 (2019).

[56] M. Freytsis, Z. Ligeti, and J. T. Ruderman, Phys. Rev. D 92 , 054018 (2015).

[57] I. Dorsner, S. Fajfer, A. Greljo, J. Kamenik, and N. Kosnik, Phys. Rep. 641, 1 (2016).

[58] M. Jung and D. M. Straub, J. High Energy Phys. 01 (2019) 009.

[59] P. Colangelo and F. De Fazio, Phys. Rev. D 95, 011701 (2017).

[60] A. Crivellin and S. Pokorski, Phys. Rev. Lett. 114, 011802 (2015).

[61] S. Faller, T. Mannel, and S. Turczyk, Phys. Rev. D 84, 014022 (2011).

[62] B. Dassinger, R. Feger, and T. Mannel, Phys. Rev. D 75, 095007 (2007).

[63] P. J. Fox, Z. Ligeti, M. Papucci, G. Perez, and M. D. Schwartz, Phys. Rev. D 78, 054008 (2008).

[64] S. Descotes-Genon, A. Falkowski, M. Fedele, M. GonzalezAlonso, and J. Virto, J. High Energy Phys. 05 (2019) 172. 